\title{
Smart Contract-based Consensus Building for Collaborative Medical Decision-Making
}

\author{
Hamza Sellak ${ }^{1}$, Mohan Baruwal Chhetri ${ }^{1}$, Zijin Huang $^{2}$, Marthie Grobler ${ }^{1}$ \\ ${ }^{1}$ CSIRO's Data61, Melbourne, Australia \\ ${ }^{2}$ The University of Sydney, Sydney, Australia \\ \{hamza.sellak, mohan.baruwalchhetri, marthie.grobler\}@data61.csiro.au, zijin.huang@sydney.edu.au
}

\begin{abstract}
Medical decision-making is moving away from the traditional one-off dyadic encounter between the patient and physician, and transitioning towards a more inclusive, shared decision-making process that also considers the inputs from other stakeholders. This ensures that a patient's decision is not only based on a medical opinion, but also includes other considerations such as impact on family members, legal and financial implications, and experiences of patients in similar situations. However, given the sensitive nature of health data and decisions, there are several challenges associated with safeguarding the privacy, security and consent of all contributors and assuring the integrity of the process. We propose a collaborative medical decision-making platform that uses a consensus building mechanism implemented using Blockchain-based Smart Contracts to address some of the above challenges, thereby giving the participants confidence that both the decision-making process and the outcome(s) can be trusted. We also present a proof-of-concept implementation using the private Ethereum Blockchain to demonstrate practicability.
\end{abstract}

\section{Introduction}

Shared decision-making (SDM) is considered the pinnacle of patient-centered care since it involves active patient engagement in making healthcare decisions that affect them directly [1]. Research has traditionally conceptualised SDM as a one-off dyadic encounter between the patient and physician within the confines of the consultation room. However, SDM is increasingly recognised as a collaborative process that involves not only the patient and physician but also other stakeholders, including family members, friends, carers, multi-disciplinary medical teams, fellow patients, social workers, and legal representatives [2]. The preferences and values of this diverse group of participants can be influenced by a range of different cultural, financial, ethical, legal, spiritual, and other determinants, and in turn shape the decision that is presented to the patient.

Consider the scenario of an early stage breast cancer patient who needs to choose between mastectomy and lumpectomy with radiation [3]. Although the patient maintains final decisional authority to accept or reject any medical intervention, she may seek input and guidance from the lead clinician, surgeon, radiation oncologist, medical oncologist, family member(s), well-wisher(s), and other fellow patient(s). However, she may not be willing to share her health data and personal preferences and values with all of them. For instance, she may not want to share her health data with certain family members, or her spiritual and cultural values with the lead clinician. Family members may not want to reveal their preferences for the different treatment options to the patient or to other family members. The members of the multi-disciplinary medical team may want to discuss their professional opinions with each other and reach a resolution before sharing their unified recommendation with the patient or the family members. Fellow patients may be willing to share their personal experiences only under the condition of anonymity.

Given the complexity of the SDM process and human perception and behaviour, it is unlikely that an agreement that considers the patient's personal preferences, healthcare providers' recommendations, and family members' wishes can be reached in a single clinical encounter. This necessitates a collaborative process that is considered reliable and trustworthy by all participants, protects sensitive information at all times, enables traceability and auditability to maintain accountability, and supports distributed, iterative, and patient-centered deliberation, extending beyond the traditional boundaries of SDM [4].

To address these challenges we propose a novel platform for collaborative medical decision-making (CMDM) that enables participants to collaboratively develop and agree on medical decisions that are 
in the best interest of the patient. In a shift from traditional face-to-face consultations, our proposed approach, which leverages Blockchain-based Smart Contract technology, supports asynchronous digital deliberation via pseudo-anonymous participation, empowering all participants to honestly share their opinions and influence the final decision. The use of Blockchain technology helps address some key concerns such as security, privacy, transparency, immutability, traceability and auditability. We demonstrate the practicability of the proposed system by implementing a proof-of-concept using the private Ethereum Blockchain. To the best of our knowledge, our proposal is the first to use a Smart Contract-based consensus reaching process for patient-centred medical decision-making.

The paper proceeds as follows. Section 2 presents related work on medical decision-making, consensus building, and Blockchain and Smart Contracts application in healthcare. Section 3 details the problem addressed in this research work while Section 4 presents our proposed solution, including a proof-of-concept implementation. Section 5 concludes the paper.

\section{Background and Related Work}

This section presents an overview of medical decision-making models, consensus building, and blockchain technology.

\subsection{Medical Decision-Making}

The research literature has traditionally considered medical decision-making to be a dyadic encounter between patient and physician [5] involving three types of decision-making models. In the paternalistic model, the physician is solely responsible for the medical decision, while the patient's participation is restricted to providing consent for the treatment. Conversely, in the informed model, the patient deliberates on the medical decision, while the physician's role is limited to that of providing expertise and information. In contrast, in SDM the physician shares information about the patient's condition and the treatment alternatives while the patient shares preferences over the different treatment alternatives, and both parties take appropriate steps to jointly reach agreement on the preferred treatment option. With the growing technologisation of healthcare and the increasing digitisation of knowledge, medical decision-making has also been considered a triadic consultation involving the computer as a third influential part [6]. There is growing consensus that medical decision-making can no longer be dyadic (or triadic) and one-off, but must involve multiple stakeholders [7]. To the best of our knowledge, our proposed CMDM platform based on iterative consensus building is the first of its kind that extends medical decision-making beyond the traditional SDM to a truly patient-centred collaborative process.

\subsection{Consensus Building}

Group decision-making (GDM) is a crucial activity of human life in which multiple participants, with individual opinions and preferences, attempt to make a collective decision regarding a specific real-life problem or situation [8]. However, those individual opinions and preferences may differ substantially, and therefore, the decision(s) resulting from their fusion might not be acceptable to all participants. A consensus building mechanism may increase the agreement level within the group prior to proceeding to the selection phase, i.e., the last step in the decision-making process, in which a selection criterion is applied to determine the group's final decision(s) [9]. Consensus building is a popular approach in the field of economics and decision science [10] that has been applied in a diverse range of application domains such as public policy [11], smart cities [12], renewable energy [13], and education [14].

As far as we know, limited research has been done on using consensus building for SDM. Enabling consensual and collectively accepted decisions is paramount to ensure that patients receive the best medical options that not only reflect their personal preferences and values but also their doctors' recommendations and families' wishes. Although a number of studies have suggested the use of generic multi-criteria decision-making techniques and/or a GDM model to help patients, physicians, and their families select a medical treatment among a set of available options [15, 16, 17], these proposals limit the decision-making process to a single round of discussion and do not guarantee that all participants' preferences are considered. In contrast, our proposal supports multi-party, iterative consensus building and also considers additional participant, process, and information-centric requirements as outlined in Section 3 and 4.

\subsection{Blockchain and Smart Contracts}

A Blockchain is an immutable, distributed ledger for recording digital transactions that are secured by cryptographic techniques and managed by a decentralized community over a peer-to-peer network [18]. Blockchain technology has a number of inherent properties that address some of the challenges of SDM including security, privacy, transparency, immutability, traceability and auditability [4]. Using 
Blockchain, complex interactions between multiple stakeholders can be documented and tracked through a time-stamped workflow. Additionally, Blockchain supports the use of pseudo-identities to retain the anonymity of participants. Smart Contracts are deterministic, immutable computer programs that are deployed and executed on the Blockchain [19]. Once deployed, they cannot be changed - the only way to modify a Smart Contract is to deploy a new instance. Therefore, Smart Contracts are protected from deletion, tampering and revision. They can be developed and run as decentralized apps that interact with the Blockchain and facilitate on-chain storage.

There are several proposals in the literature on Blockchain-based healthcare applications including health data sharing [20], patient health records [21], clinical trials [22], and wearable data marketplace [23]. To the best of our knowledge, there is only one previous research work that uses Blockchain technology for CMDM using the notion of Proof-of-Familiarity [24]. However, it limits the clinical encounter to a single round making it impractical in a real-world setting.

\section{Problem Statement}

Consensus building to identify the best possible medical intervention for the patient may not be possible in a face-to-face setting due to several reasons. The group decision might be unduly influenced and biased by more dominant individuals or subgroups; participants may refuse to honestly share their preferences, values and opinions with the group; and the face-to-face discussion may limit patients' ability to make decisions based on shared inputs. In this section, we first provide an overview of a consensus reaching process for CMDM following by a discussion of the key participant, process and information-centric requirements associated with consensus building for CMDM.

\subsection{Framework}

The key elements of the proposed consensus reaching process are described as follows.

- $T=\left\{t_{1}, t_{2}, \ldots, t_{m}\right\}$ denotes a finite set of $m(\geq 2)$ treatment options available to the patient, e.g., $t_{1}$ : mastectomy and $t_{2}$ : lumpectomy (with radiation).

- $C=\left\{c_{1}, c_{2}, \ldots, c_{n}\right\}$ denotes a finite set of $n(\geq 2)$ criteria that are considered in the evaluation of the treatment options, e.g., $c_{1}$ : effectiveness, $c_{2}$ : side effects, $c_{3}$ : survival rate, $c_{4}$ : invasiveness, $c_{5}$ : recovery time, and $c_{6}$ : related costs.

- $P=\left\{p_{1}, p_{2}, \ldots, p_{o}\right\}$ denotes a group of $o(\geq 2)$ participants, e.g., $p_{1}$ : patient, $p_{2}$ : partner, $p_{3}$ : lead clinician, $p_{4}$ : multi-disciplinary cancer team, $p_{5}$ : fellow patients, and $p_{6}$ : insurance company 1 .

- $\Lambda_{(r)}=\left(\lambda_{1(r)}, \lambda_{2(r)}, \ldots, \lambda_{o(r)}\right)^{T}$ denotes a weight vector representing the influence of all participants in a given consensus round $r$, such that $\lambda_{k(r)}$ is the influence of participant $p_{k} \in P$ and $\forall p_{k} \in$ $P, 0 \leq \lambda_{k(r)} \leq 1$ and $\sum_{k=1}^{o} \lambda_{k}=1$, e.g., $\Lambda_{(1)}=(0.5,0.05,0.2,0.2,0.025,0.025)^{T}$ is the initial weight vector assigned to the group of participants $P$ in which the patient is given higher weight (influence) in comparison to the rest of the participants.

- Each participant $p_{k} \in P$ provides his/her opinion over $T \times C$ in a given consensus round $r$ by means of an $m \times n$ decision matrix $\left(D M_{k(r)}\right)$ :

$$
D M_{k(r)}=\left(\begin{array}{ccc}
d m_{k(r)}^{11} & \ldots & d m_{k(r)}^{1 n} \\
\vdots & \ddots & \vdots \\
d m_{k(r)}^{m 1} & \ldots & d m_{k(r)}^{m n}
\end{array}\right)
$$

where each assessment $d m_{k(r)}^{i j}$ indicates the degree to which the $i^{\text {th }}$ treatment option satisfies the $j^{\text {th }}$ criterion according to the $k^{t h}$ participant in a given consensus round $r$. It can be expressed using an appropriate information domain - numerical values or linguistic terms - and a specific preference structure such as intervals, n-tuple or fuzzy sets.

- Figure 1 shows the automated consensus builder that replaces the human mediator by following the general scheme of a consensus reaching process [9]. Firstly, individual preferences of the participants are combined using an appropriate aggregation operator and the current consensus degree, i.e., level of agreement within the group, $C D_{c(r)}$ is computed using a predefined consensus measure. The consensus degree is then compared with a preset minimum consensus threshold. If the consensus degree exceeds the threshold $\left(C D_{c(r)}>\right.$ threshold $)$, the group moves on to the selection phase; otherwise, the consensus builder takes appropriate steps to increase the level of agreement in the following consensus round. This can be done in different ways including through an interactive feedback process [13] and automatic adjustment of the participants' preferences [25]. Consensus must be achieved before a maximum predefined number of iterations $r_{\max }$ is surpassed, i.e., the process continues until $C D_{c(r)} \geq$ threshold or $r>r_{\max }$.

\footnotetext{
${ }^{1}$ For simplicity, we represent the multi-disciplinary cancer team and the group of former breast cancer patients as single entities.
} 


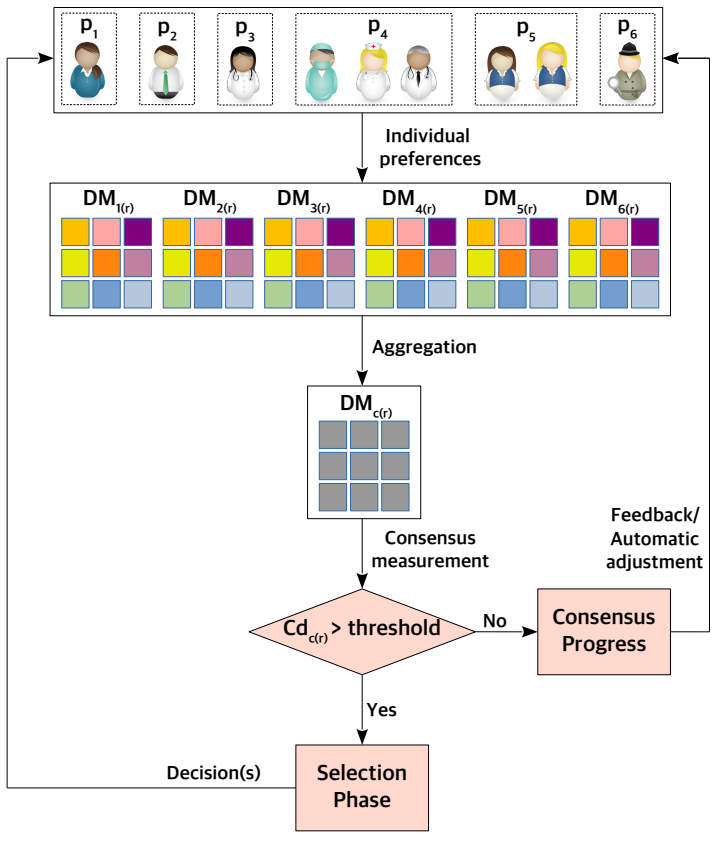

Figure 1. Automated consensus builder.

\subsection{Process Initiation and Participation}

This section describes how the CMDM process may be initiated by select participants, and how the remaining participants engage by invitation only.

- As the main stakeholder, the patient $\left(p_{1}\right)$ may initiate the process to discuss the different treatment options $\left(t_{1}\right.$ and $\left.t_{2}\right)$ with the group $P$ or to seek a second/third opinion on her condition and available/recommended treatment options. In both scenarios, the patient may either participate directly in the CMDM process and ensure that her preferences are considered in reaching consensus, or wait for the final outcome of the process and make an informed decision after reviewing the group's recommendation on $t_{1}$ and $t_{2}$.

- The partner $\left(p_{2}\right)$ (family member/s or legal representative) may initiate the process on behalf of the patient if she is legally, physically, and/or mentally incapable of making her own decisions, or if explicitly asked by the patient to help reach a decision by collaborating with the other stakeholders. In either case, they have two options available to them - either directly participate in the CMDM process or wait for the final group recommendation.

- The lead clinician $\left(p_{3}\right)$ or the multi-disciplinary cancer team $\left(p_{4}\right)$ may initiate the process to seek further opinions from other health professionals, either due to low clinical evidence, to resolve disagreements, justify/confirm their recommendations, or share accountability. They may decide to directly participate in the CMDM process or wait for the consulted experts' feedback and inform the patient about the recommendations, along with their own professional opinion.

- The insurance company, which is concerned with the treatment costs, may demand further opinions from its trusted health professionals before financially approving any medical interventions. The insurance agent $\left(p_{6}\right)$ may decide to represent the company in the CMDM process and directly participate by explicitly expressing their support/non-support of each available treatment option or wait for the group's recommendations and then inform the patient whether they can or cannot be covered by the insurance policy.

\subsection{Key Requirements}

This section describes a number of participant, process and information-centric requirements that the CMDM process has to satisfy to ensure that all participants are fully supported.

\subsubsection{Participant-Centric Requirements (ParRQs)} Fulfilling the following requirements may encourage participation by the different types of stakeholders.

- Autonomy - Participants should have the opportunity to exercise freedom of thought and free will and make informed, unconditional, and uncoerced decisions independent of other participants' viewpoints. For instance, the patient should have the freedom to choose whether or not to reveal her spiritual or cultural beliefs, and to whom.

- Transparency - Participants should have a clear understanding of their right and obligations, and the benefits and potential risks of participating in the CMDM process. For instance, an external healthcare professional might want clear communication about the legal obligations of his/her participation and negotiate them if needed. Participants should also have the right to understand how their opinions and views are collected, recorded, and stored, and when and how this information is used and shared.

- Anonymity/Pseudonymity - Participants should have the right to participate in the CMDM process and express their true preferences and opinions without fear or favour. Participants should also be able to selectively reveal their identity to some or all of the participants [26]. For instance, some fellow patients might agree to participate only if they can use pseudonyms and cannot be identified by the other 
participants as this might disclose very sensitive information such as the fact that they had or still have breast cancer and experienced the side effects of some of the treatment options.

- Privacy - Participants should be able to control how their personal information (including health information, and personal preferences, opinions and views) is shared with others. For instance, some family members may want to grant the patient access to their preferences for the different treatment options but restrict access to other family members.

\subsubsection{Process-Centric Requirements (ProRQs)} Participants may not contribute honestly to the CMDM process if they do not consider it reliable and trustworthy. Satisfying the following ProRQs may help address this.

- Authenticity - The CMDM process should ensure that only authenticated participants contribute to decision-making and that the input data originates from its purported source [27]. Knowing that the authenticity of the participants is verified during all stages of the CMDM process and providing confirmation that the input data can be trusted will give the patient confidence in the validity of outcomes.

- Accessibility - The CMDM process should allow digital deliberation and collaboration among geographically dispersed participants through verified asynchronous communication and authorised sharing of preferences, opinions, and ideas. For instance, a patient located in Melbourne may want to invite an oncologist and surgeon who are in Sydney and Brisbane, respectively, to participate in the CMDM process along with some fellow patients who are potentially located in other countries.

- Automated Mediation - The CMDM process should proceed with minimum or zero human moderation. The participant who initiates the process should be able to define some parameters such as the minimum consensus (threshold) and maximum number of iterations $\left(r_{\max }\right)$, but an automated consensus builder should orchestrate the consensus reaching process leading to an outcome. Knowing that the execution of the process is delegated to a trusted mediator to minimise (human) error and bias might increase the participants' engagement with the CMDM process.

- Reliability - Participants should have assurances about the integrity of the CMDM process. The process should be complete (i.e., cover all possible scenarios), consistent (i.e., always return the same outcome for a given process configuration and the same set of inputs from all participants in each round of the interaction), and accurate (i.e., return results that are accurate as per the encoded consensus building logic). For instance, if there is randomness in the consensus building mechanism this will lead to the participants questioning the quality of the outcomes as running the process with the same decision matrices might lead to different (better) decisions. Randomness is not tolerated in systems that generate high-stakes decisions and require non-repudiation of any kind.

\subsubsection{Information-Centric Requirements (InfRQs)} Participants may abstain from contributing truthfully to the CMDM process if they feel that their information will not be kept private and secure. Satisfying the following set of InfRQs may increase participants' trust in the process [28].

- Confidentiality - The CMDM process should guarantee that participants' personal information is kept confidential at all times, i.e., it is only shared with authorized entities based on the participants' explicit consent. For instance, doctor-patient confidentiality is a legal privilege granted to patients [29].

- Integrity - The information collected during the CMDM process should be maintained in a correct state at all times. Nobody should be able to modify the participants' decision matrices, either accidentally or maliciously. If a participant decides to leave the CMDM process before consensus is reached or the process has terminated, all of their data should be treated as per prior agreement, i.e., it should be destroyed or used anonymously but never modified to bias or accelerate the consensus reaching process.

- Availability - Information should be made available when requested by authorised entities. For instance, when a participant wants to check their decision matrix during a previous consensus round or when the CMDM process needs to operate on individual decision matrices at the end of each round to generate the appropriate feedback to be sent to each participant.

- Auditability - At the end of the CMDM process, the patient and/or lead clinician might want to conduct a comprehensive examination of the process and understand how the group achieved a decision without violating the participants' anonymity.

- Non-repudiation - The CMDM process should be able to confirm the occurrence/non-occurrence of an action. This is important to give assurance that the sender of a decision matrix or (any information) is provided with proof of delivery and the consensus builder is provided with proof of the sender's identity, so neither can later deny the data exchange [27]. 
- Trustworthiness - The CMDM process should be able to verify identity and establish trust in all participants. It should verify attributes of a participant so that the patient has confidence in their qualifications, capabilities, and ability to contribute to the decision and fulfill assigned responsibilities.

- Privacy - The CMDM process needs to ensure that privacy protections are implemented in the collection, use, sharing, storage, transmittal, and disposal of information that each participants should have control over their personal information.

The ParRQs, ProcRQs, and InfRQs should be collectively satisfied as they are complementary and will not be effective if considered individually. For example, if the CMDM process satisfies the ProcRQs and InfRQs but not the ParRQs, this might result in abstention from participation regardless of whether it is promoted as reliable, trustworthy and information-secure. Therefore, innovative mechanisms should be designed to ensure that these requirements are satisfied to give all participants confidence that the proposed CMDM approach can replace the traditional SDM.

\section{Proposed Platform}

This section presents the high-level conceptual architecture for the proposed platform and a prototype implementation using the Ethereum ${ }^{2}$ blockchain. In practice, the blockchain would be run by a consortium of hospitals and insurance companies such that no one single institution controls the network and participants would be able to register with the platform using appropriate mechanisms provided by the consortium.

\subsection{Conceptual Architecture}

Figure 2 depicts the high-level conceptual architecture for the proposed CMDM platform. We propose five stages for consensus building and discuss them using the running example of an early breast cancer patient $p_{1}$ who has to choose between two treatment options $t_{1}$ and $t_{2}$. We consider a scenario where $p_{1}$ does not directly participate in the CMDM process, but instead makes a decision after reviewing the recommendations from her partner $p_{2}$, the lead clinician $p_{3}$, the multi-disciplinary cancer team $p_{4}$, fellow patients $p_{5}$, and the insurance company $p_{6}$. The Consensus Builder is responsible for orchestrating the CMDM process.

- Step 1. The platform sends invitations to participants on behalf of the patient. The invitation should include

\footnotetext{
${ }^{2}$ https://ethereum.org/en/
}

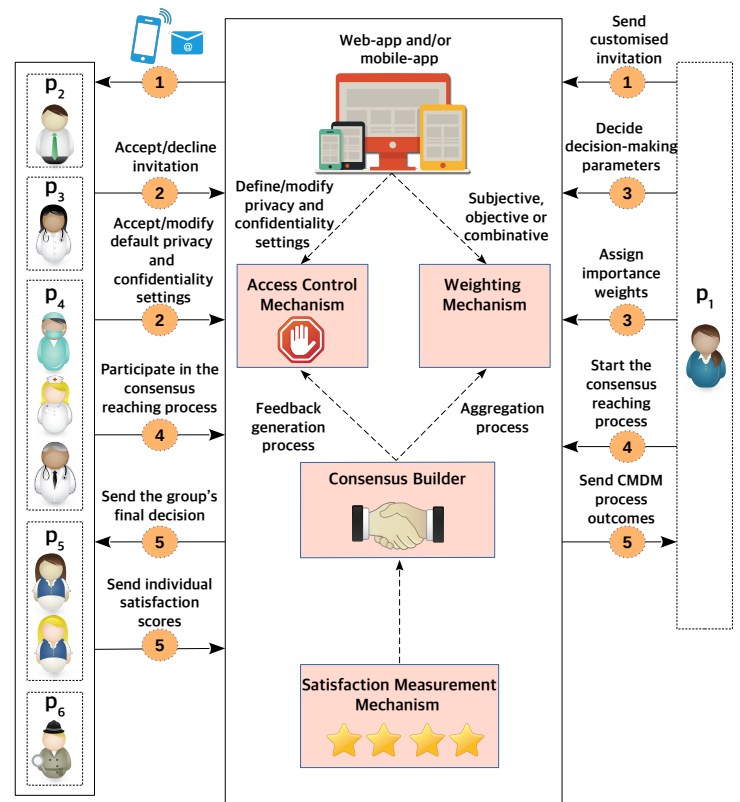

Figure 2. High-level architecture of the patient-centred CMDM platform.

the terms and conditions of participation, and may contain details about the patient (if not anonymous), their medical condition, the invitee's obligations as a participant, details about other participants (if not anonymous), liability (if any), etc. The invitation should also provide an accessible link to the platform.

- Step 2. Participants accept or decline the invitation, or ask for further information. As part of the invitation, participants receive the predefined privacy and confidentiality settings, either explicitly defined by the patient, or automatically generated by the platform using an appropriate access control mechanism. They can modify the settings that apply to them at any point during the process. Examples include identity management to hide/reveal their real identity using anonymisation/pseudo-anonymisation techniques, and preference sharing rules to control who can access their preferences (decision matrix). For the purpose of illustration, we assume that all participants in our scenario accept the invitation to join the process after defining their privacy and confidentiality settings.

- Step 3. The patient defines the decision-making parameters (i.e., treatment options, evaluation criteria, consensus threshold, and maximum number of consensus rounds) or asks for everyone's involvement to collectively identify them. The patient can decide if she wants all participants to evaluate the same treatment options using the same set of criteria, or specify a different set for each participant based on 
their profiles and expected contribution. For instance, the medical team can advise on the treatment options according to $c_{1}$ : effectiveness, $c_{2}$ : side effects, $c_{3}$ : survival rate, $c_{4}$ : invasiveness, and $c_{5}$ : recovery time, whereas the insurance company can only advise on $c_{6}$ : related costs. The patient can use the weighting mechanism to assign importance weights to each participant's contribution subjectively, objectively or using some combination of the two.

- Step 4. The patient triggers the Consensus Builder once she is satisfied with the set of participants $P$ and their importance weights, and all participants have defined their privacy and confidentiality settings and agreed to the decision-making parameters. The iterative consensus reaching process starts without any further intervention from the patient by following the steps depicted in Figure 1. A fixed deadline, defined based on the criticality of the patient's condition, may be applied to each round to prevent delays and ensure positive progression of the CMDM process.

- Step 5. An overall score is computed for the different alternatives, $t_{1}$ and $t_{2}$ in our scenario, based on the group's last collective decision matrix. Using the satisfaction measurement mechanism, participants are then explicitly asked to indicate how satisfied they are with the group's final decision. These individual scores, along with other relevant information such as consensus degree, number of consensus rounds needed to reach consensus (if applicable), and any additional comments the participants want to share with the patient, form part of the outcome sent to the patient for consideration. The satisfaction measurement is intended to increase the patient's confidence in the group decision.

\subsection{Prototype Implementation}

Figure 3 shows our proof-of-concept CMDM platform implementation. The main CMDM process is realised via two Smart Contracts - Consensus Builder Contract and Access Control Contract. The automated Consensus Builder, Weighting Mechanism, and Satisfaction Measurement Mechanism are encoded in the Consensus Builder Smart Contract. Similarly, the Access Control Mechanism, which enables controlled access to the information exchanged during the process based on the participants' preferences, is encoded in the Access Control Smart Contract.

All interactions between the participants and the platform happen via the API gateway. Participants would need to first register themselves with the platform. We omit the details on how this would occur due to space limitations. Once they have registered, participants can participate in one or more CMDM

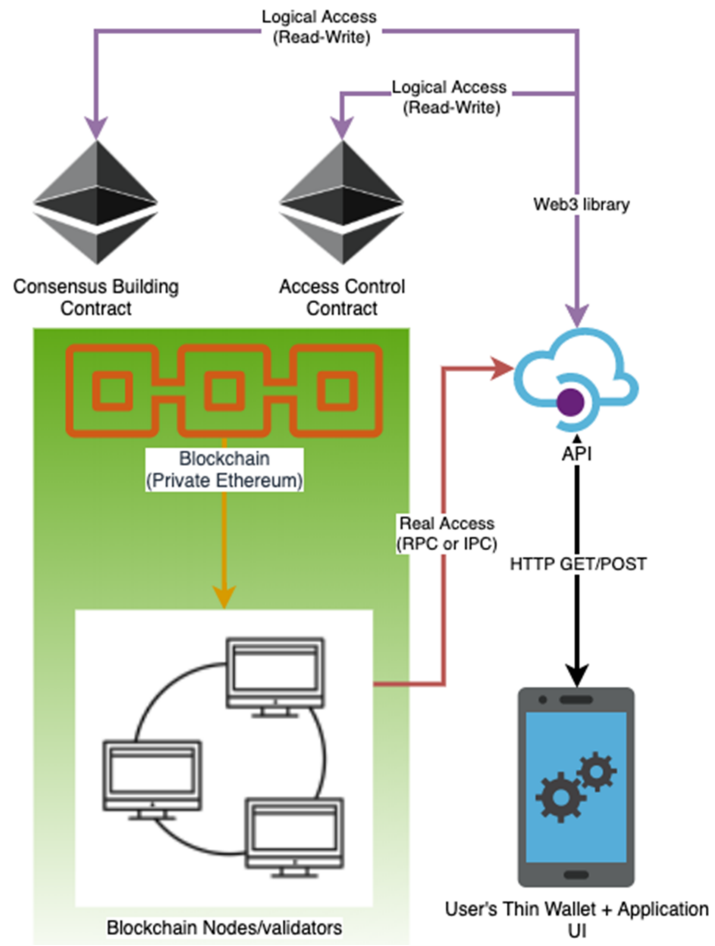

Figure 3. Technical architecture of the patient-centred CMDM platform.

process instances. In order to interact with the Smart Contracts on the Ethereum Blockchain, all participants need to have their own private keys that can be obtained by registering with any Ethereum wallet provider.

Both Smart Contracts, written in Solidity ${ }^{3}$, are deployed on the Ethereum blockchain. The Web3.py library is used to interact with the two Smart Contracts, parse private keys into account addresses, and to lodge transactions. We use Truffle 5 as the development framework and Ganache 6 a local emulator of the Ethereum Blockchain, for testing the prototype. We used the keys provided by Ganache.

4.2.1. CMDM Orchestration Figure 4 illustrates how the different participants interact with the two Smart Contracts. We use the scenario described in Section 4.1 to demonstrate how the prototype works and the steps described below loosely correspond to the steps outlined in Section 4.1 .

- Step 1. The patient initiates the process and configures the required parameters including questions related to the treatment options, consensus threshold,

\footnotetext{
${ }^{3} \mathrm{https}$ ://docs.soliditylang.org/en/v0.8.3/

${ }^{4}$ https://web3py.readthedocs.io/en/stable/

${ }^{5}$ https://www.trufflesuite.com/truffle

${ }^{6} \mathrm{https}: / /$ www.trufflesuite.com/ganache
} 


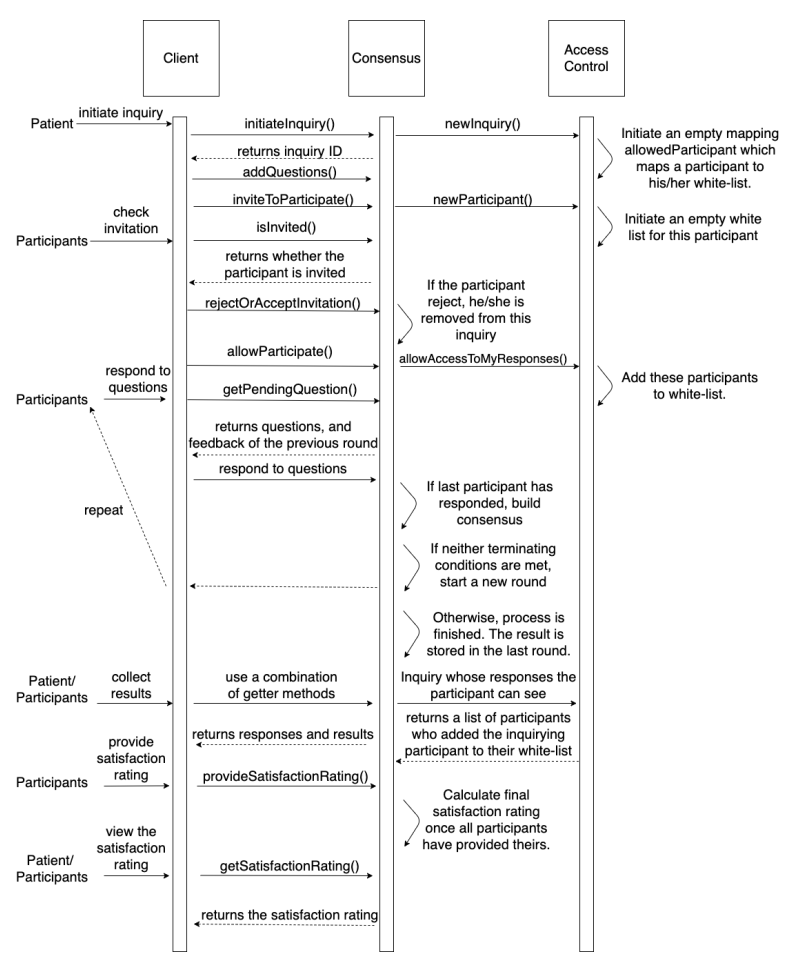

Figure 4. Sequence diagram of participants' interaction with Consensus and AccessControl.

and maximum number of consensus rounds. The patient then adds the list of invited participants, and configures the initial privacy settings. This is illustrated in Figure 5

- Step 2. Selected participants receive notification of the invitation and can choose to accept or decline the invitation. Participants that accept the invitation are required to specify who can see their responses, provided the patient has allowed participants to selectively allow access to their responses when configuring the process in the previous step. Once all participants have accepted/declined their invitation, the consensus reaching process can begin.

- Step 3. In each consensus round, participants retrieve questions from the Smart Contract and respond to them based on their personal preferences. This is illustrated in Figure 6. Depending on how access control has been configured, they may/may not be allowed to view the individual responses/group aggregated response in the previous round.

- Step 4. Once all participants have provided their responses, the Consensus Builder aggregates the results for the current round and calculates the consensus degree. If the consensus degree exceeds the threshold or the maximum number of rounds has been reached, the process proceeds to Step 5, otherwise

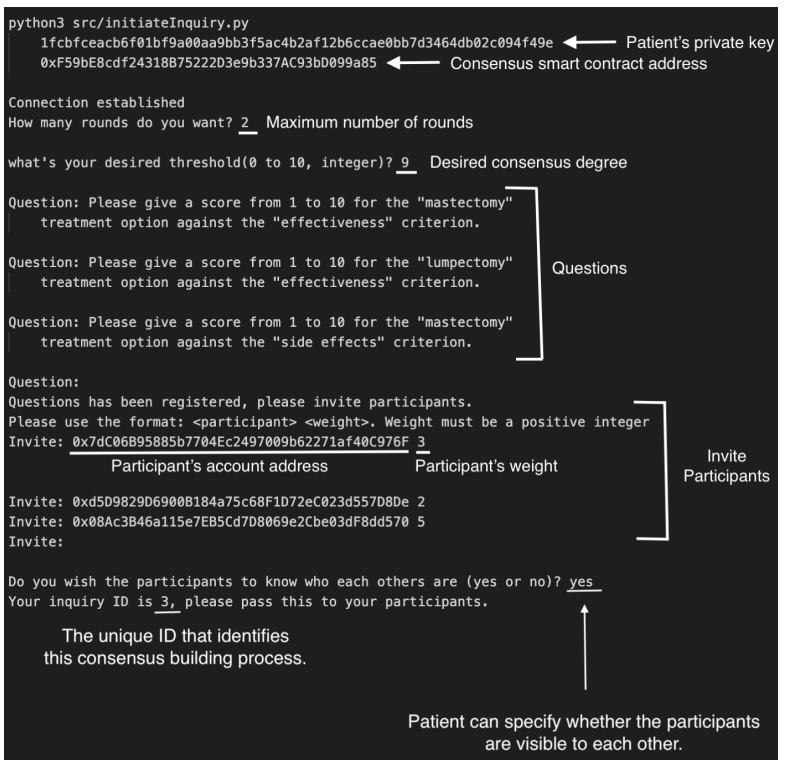

Figure 5. Patient initiates a new process.

each participant is provided with a personalised feedback that can help them align their responses with the rest of the group in the next round and Steps 3 and $\mathbf{4}$ are repeated.

- Step 5. The patient and all participants receive the group decision as illustrated in Figure 7 . Note that the returned result will be different for each participant depending on how access control has been configured for them.

- Step 6. As a final step, all participants provide an indication of how satisfied they are with the group's decision and the process outcome is sent to the patient.

\subsection{Discussion}

Our proof-of-concept prototype implementation has demonstrated the practicability of the proposed patient-centered CMDM platform. Some of the requirements identified in Section 3.3 are met by virtue of the consensus reaching process design. The use of a digital platform ensures that participants have better accessibility. In the absence of face-to-face interactions, participants may feel more empowered and honest in sharing their personal preferences and opinions. The consensus reaching process aims to be transparent by design so that participants have a clear understanding of their rights and obligations. It supports iterative decision-making and fine-grained access control over who can access each piece of information. The use of automated mediation helps increase trust in the process by minimising human error and bias. The consensus reaching process balances 


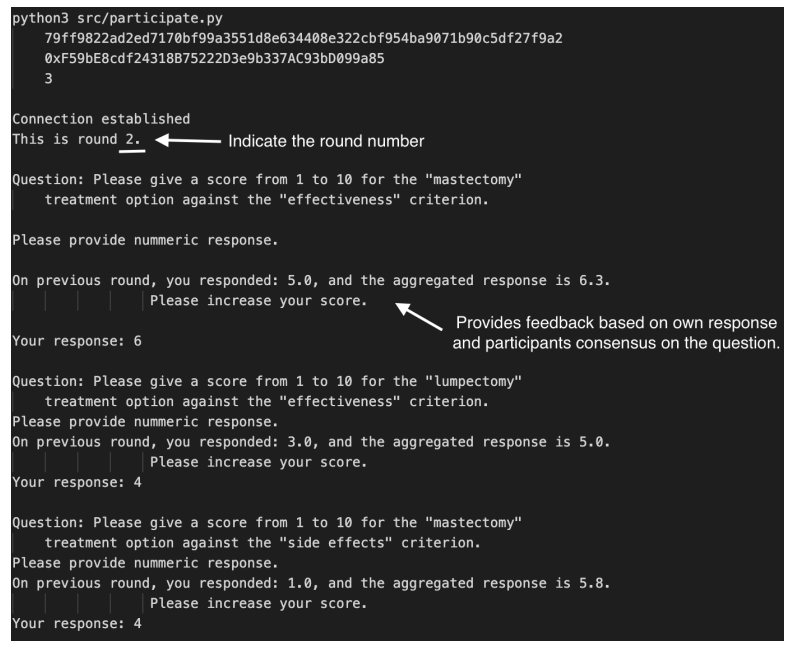

Figure 6. Participant's response to the questions.

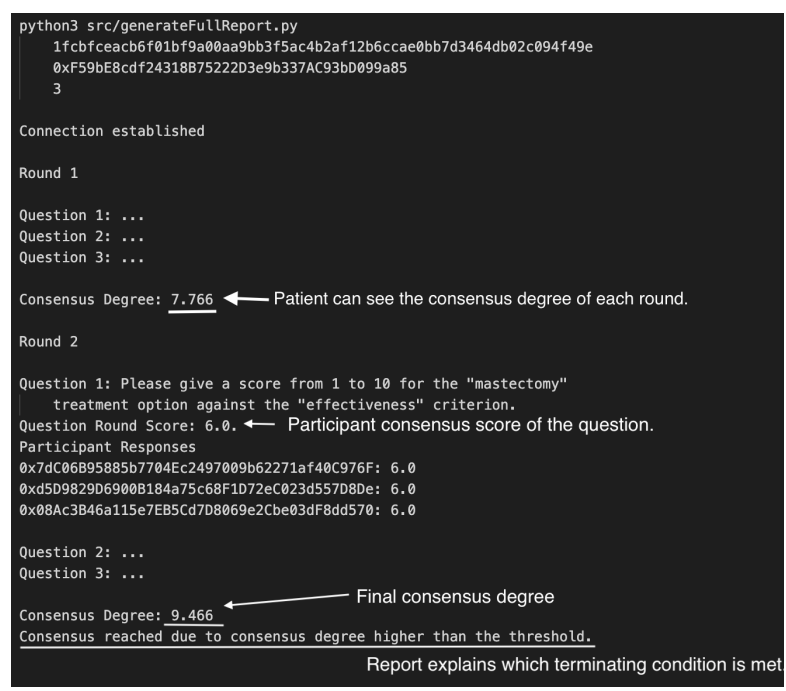

Figure 7. Report showing part of the final result.

both medical (practitioners' expertise) and supporting perspectives (family members' wishes and fellow patients' experiences) to ensure that patients (or their representatives) can make a fully informed decision. The ultimate goal of the CMDM process is to assist the patient in figuring out which treatment option aligns best with her personal preferences and values. Thus, giving the patient (or their representatives) the option to manually assign importance weights to each participant, and hence decide the influence they should have during the consensus reaching process (e.g., based on expertise or social trust), guarantees that the process and the outcome(s) are truly patient-centred. For instance, the patient may give a bigger weight to the lead clinician, multi-disciplinary cancer team, and fellow patients to ensure that the final decision is backed by medical expertise and real-life experience rather than being driven by the family members' wishes.

Using Smart Contracts to implement the consensus reaching process further helps address additional requirements identified in Section 3.3 Smart Contracts are deterministic and immutable computer programs that are stored, executed and verified on the Blockchain. Once deployed, a Smart Contract's code cannot be changed, even by the authors themselves. Encoding the consensus reaching business logic on the Smart Contract guarantees the integrity of the process. Similarly, all interactions (and associated information) between the consensus builder and the participants occur through the Consensus Smart Contract and get recorded on the Blockchain. Recording interactions on the Blockchain ensures integrity of the information as well as traceability, auditability and non-repudiability, in turn ensuring accountability in the decision-making process. The use of Blockchain also enables regulatory oversight or evidence for legal remedies in the case of negligence or undesirable outcomes resulting from the medical intervention. The use of pseudonyms enables participants to keep their identity hidden in the CMDM process with some level of anonymity while allowing the determination of identity if required. Implementing the access control mechanism using Smart Contract ensures that only authorised participants have access to the interaction data based on the allowed permissions.

However, additional support is required for ensuring full privacy and confidentiality of the data that is written on the blockchain. One way to do this is by encrypting the data before it is written on the blockchain using symmetric or assymetric encryption. An alternative way is to store the data and decisions exchanged during the CMDM process off-chain in an encrypted database and include a hash of the data on the Blockchain. This requires reconsideration of the consensus builder implementation architecture.

\section{Conclusion}

Truly patient-centered care should not be a one-off dyadic encounter between the patient and physician, but it should also involve other stakeholders including family members, carers, and fellow patients. In this paper, we proposed a novel platform for collaborative medical decision-making (CMDM) that relies on a patient-centred consensus reaching process, and allows patients to initiate a collaboration that extends beyond the traditional shared decision-making model to include both medical and non-medical perspectives in selecting an appropriate treatment option. Our solution leverages Blockchain-based Smart Contracts to address some of 
the key requirements of CMDM including ensuring accessibility, accountability, confidentiality, privacy, and transparency. We implemented a proof-of-concept prototype using the private Ethereum Blockchain to demonstrate practicability from a technical perspective. As future work, we plan to conduct studies with actual stakeholders to evaluate acceptability of the proposed solution, and also look at implementation strategies to ensure enhanced data protection.

Acknowledgement: The authors would like to thank Dr Dimaz Wijaya and Dr Sushmita Ruj for contributing to the technical architecture of the platform. Zijin Zhang was supported by a Data61 Summer Vacation Scholarship. This research was financially supported by CSIRO's Precision Health Future Science Platform.

\section{References}

[1] M. J. Barry and S. Edgman-Levitan, "Shared decision making - the pinnacle patient-centered care.," New England Journal of Medicine, 2012.

[2] J. Hamann and S. Heres, "Why and how family caregivers should participate in shared decision making in mental health," Psychiatric Services, vol. 70, no. 5, pp. 418-421, 2019.

[3] S. N. Whitney, A. L. McGuire, and L. B. McCullough, "A typology of shared decision making, informed consent, and simple consent," Annals of Internal Medicine, vol. 140, no. 1, pp. 54-59, 2004.

[4] H. Sellak, M. Baruwal Chhetri, and M. Grobler, "Towards a trusted collaborative medical decision-making platform," in International Conference on Collaborative Computing: Networking, Applications and Worksharing, pp. 407-418, Springer, 2020.

[5] E. J. Emanuel and L. L. Emanuel, "Four models of the physician-patient relationship," JAMA, vol. 267, no. 16, pp. 2221-2226, 1992.

[6] L. G. Bussey and E. Sillence, "The role of internet resources in health decision-making: A qualitative study," Digital health, vol. 5, 2019.

[7] G. Elwyn, "Arriving at the postmodern medical consultation," The European Journal of General Practice, vol. 10, no. 3, pp.93-97, 2004.

[8] J. Kacprzyk, M. Fedrizzi, and H. Nurmi, "Group decision making and consensus under fuzzy preferences and fuzzy majority," Fuzzy Sets and Systems, vol. 49, pp. 21-31, Jul 1992.

[9] F. Herrera, E. Herrera-Viedma, et al., "A model of consensus in group decision making under linguistic assessments," Fuzzy Sets and Systems, vol. 78, no. 1, pp. 73-87, 1996.

[10] Y. Dong, J. Xu, and Dong, Consensus building in group decision making. Springer, 2015.

[11] I. Pérez, P. Garcia-Sanchez, F. Cabrerizo, and E. Herrera-Viedma, "An approach toward a feedback mechanism for consensus reaching processes using gamification to increase the experts' experience," in 53rd Hawaii International Conference on System Sciences, 2020.

[12] F. Mata, A. Verdejo, L. Pérez, and C. Porcel, "A preliminary study of a citizen participation system based on consensus for decision-making processes," in 54th Hawaii International Conference on System Sciences,
2021.

[13] H. Sellak, B. Ouhbi, B. Frikh, and B. Ikken, "Expertise-based consensus building for mcgdm with hesitant fuzzy linguistic information," Information Fusion, vol. 50, pp. 54-70, 2019.

[14] F. J. Cabrerizo, I. J. Pérez, J. A. Morente-Molinera, S. Alonso, and E. Herrera-Viedma, "An adaptive feedback mechanism for consensus reaching processes based on individuals' credibility," in 52nd Hawaii International Conference on System Sciences, 2019.

[15] J. Dowie, M. Kjer Kaltoft, G. Salkeld, and M. Cunich, "Towards generic online multicriteria decision support in patient-centred health care," Health Expectations, vol. 18 , no. 5, pp. 689-702, 2015

[16] J. G. Dolan, E. Boohaker, J. Allison, and T. F. Imperiale, "Can streamlined multicriteria decision analysis be used to implement shared decision making for colorectal cancer screening?" Medical Decision Making, vol. 34, no. 6, pp. 746-755, 2014.

[17] T.-Y. Chen, "An interactive method for multiple criteria group decision analysis based on interval type-2 fuzzy sets and its application to medical decision making," Fuzzy Optimization and Decision Making, vol. 12, pp. 323-356, Mar 2013.

[18] I. Marco and K. R. Lakhani, "The truth about blockchain," Harvard Business Review, vol. 95, no. 1, pp. 118-127, 2017.

[19] M. Schnitzbauer, "Smart contracts in healthcare," Digitalization in Healthcare, p. 211, 2021.

[20] M. Mettler, "Blockchain technology in healthcare: The revolution starts here," in 18th International Conference on e-Health Networking, Applications and Services, pp. 1-3, 2016.

[21] A. Roehrs, C. A. Da Costa, and R. da Rosa Righi, "Omniphr: A distributed architecture model to integrate personal health records," Journal of Biomedical Informatics, vol. 71, pp. 70-81, 2017.

[22] Z. Shae and J. J. Tsai, "On the design of a blockchain platform for clinical trial and precision medicine," in 37th International Conference on Distributed Computing Systems, pp. 1972-1980, 2017.

[23] A. Colman, M. J. M. Chowdhury, and M. Baruwal Chhetri, "Towards a trusted marketplace for wearable data," in 5th International Conference on Collaboration and Internet Computing, pp. 314-321, 2019.

[24] J. Yang, M. M. H. Onik, N.-Y. Lee, M. Ahmed, and C.-S. Kim, "Proof-of-familiarity: A privacy-preserved blockchain scheme for collaborative medical decision-making," Applied Sciences, vol. 9, no. 7, p. 1370, 2019.

[25] I. Palomares, H. Sellak, B. Ouhbi, and B. Frikh, "Adaptive semi-supervised consensus model for multi-criteria large group decision making in a linguistic setting," in 12th International Conference on Intelligent Systems and Knowledge Engineering, IEEE, Nov 2017.

[26] "Australian Privacy Principles Guidelines: APP 2-Anonymity and pseudonymity." https: //tinyurl.com/oaic-privacy, 2019

[27] "Computer Security Resource Center Glossary." https://csrc.nist.gov/glossary/, 2019.

[28] Y. Cherdantseva and J. Hilton, "A reference model of information assurance \& security," in 2013 International Conference on Availability, Reliability and Security, pp. 546-555, IEEE, 2013.

[29] "Confidentiality and privacy in healthcare." https:// tinyurl.com/yv3s5t5t 2015. 